\title{
Common Agency and the Revelation Prindiple
}

\author{
Michael Peters ${ }^{\alpha}$ \\ Department of Economics \\ University of Toronto \\ 150 St. George St. \\ Toronto, Canada \\ M5S 367 \\ J anuary 19, 1999
}

\begin{abstract}
A bstract
In the common agency problem multiple mechanism designers simultaneously attempt to control the behavior of a single privately informed agent. The paper shows that the allocations associated with equilibria relative to any ad hoc set of feasible mechanisms can be reproduced as equilibria relative to (some subset of) the set of menus. Furthermore, equilibria relative to the set of menus are weakly robust in the sense that it is possible to ${ }^{-}$nd continuation equilibria so that the equilibrium allocations persist even when the set of feasible mechanisms is enlarged.
\end{abstract}

The set of direct mechanisms ${ }^{1}$ forms a universal dass of medhanisms for problems in which a single principal tries to control the incentives of one or more agents. These mechanisms are universal in the sense that the payo ${ }^{R S}$ (or allocations) generated by any indirect mechanism can be reproduced by

\footnotetext{
${ }^{x}$ Thanks are due to Larry Epstein, Meg Meyer, Carolyn Pitchik, Lars Stole and Sergei Severinov for helpful comments. The ${ }^{-}$nancial support of the Social Sciences and Humanities Research Council of Canada is gratefully adknowledged.

${ }^{1}$ Early references include Gibbard [5], Green and Laßont [6], and Myerson [10].
} 
an appropriatedirect mechanism. An immediateimplication is that the most pro table direct mechanism is always at least as pro table as any indirect mechanism. In this sense, optimal direct medhanisms are robust in the sense that sellers will continue to use them even if the set of feasible mechanisms is enlarged.

It has been known for sometimethat 'direct' mechanisms of the usual sort do not have these properties when there are multiple principals competing against one another. ${ }^{2}$ The di \pm culty is that agents have market information at the time that they communicate with principals and 'naive' direct mechanisms do not allow sellers to use this information. Alternatively, a complete description of theagent's preferences (and beliefs) is not enough to reveal the agent's 'type' in the sense of Harsanyi. Examples in the literature show that equilibrium in direct mechanisms is not robust against the possibility that the mechanism designers might deviate outside this class of mechanisms, ${ }^{3}$ and that there are dasses of indirect mechanisms whose equilibrium allocations cannot be replicated when sellers are compelled to use only direct mechanisms ${ }^{4}$.

At a conceptual leve, this problem has been resolved by Epstein and Peters [4] who construct a universal set of mechanisms based, in part, on a rede- nition of an agent's type. This set of mechanisms has both the de sirable properties that the set of direct mechanisms possesses in the usual single principle problem. First, any indirect model of competition among mechanism designers gives rise to a set of feasible mechanisms that can be embedded into the universal set of mechanisms. Secondly, equilibria relative to the universal set of mechanisms are robust in the sense that equilibria will continue as equilibria when the set of feasible medhanisms is extended.

The set of types involved in such a construction are complex. First of all, sincetype contains market information, it is endogenous. Second, even in single agent environments where the principals' beliefs are common knowledge,

\footnotetext{
${ }^{2}$ The basic problem with direct mechanisms in competing mechanism design problems was ${ }^{-}$rst observed by McAfe [9] and Katz [7]. Examples illustrating the di \pm culties that arise are given in [8], [14], and [4]

${ }^{3}$ An example is given in Martimort and Stole [8].

${ }^{4}$ Martimort and Stole [8] give an equilibrium in which sellers use reasonable looking indirect mechanisms. Peck [14] gives an example of a non-truthful equilibrium in direct mechanisms whose allocations cannot be replicated in any equilibrium in which sellers use direct mechanisms.
} 
the principals' mechanisms can depend on one another in complex ways. For example, seller 1's mechanism may depend on whether seller 2's mechanism depends on whether seller 1's mechanism depends :: : and so on. So the set of agent's types must come from an in ${ }^{-}$nite dimensional space even when the set of agent's types is ' nite in the conventional sense So despite the fact that a universal set of mechanisms is known to exist, it is di \pm cult to use this result in practice.

The purpose of this paper is to ask whether the construction of a universal set of mechanisms is simpler in a more special ized environment. The natural candidate for such an investigation is that class of problems where multiple principals attempt to control the incentive of a single agent. This model has been the focus of the emerging literature on common agency. ${ }^{5}$ One of the methods that has been used to analyze problems in this framework is to imagine that the principals o eer the agent menus of alternative from which he can choose In the single mechanism designer problem with a single agent who has private information but takes no unobservable action, menus of alternatives are clearly equivalent to direct mechanisms ${ }^{6}$. This is true no matter how complicated thetype space is, any contract that asks the agent to report a type is o eering the agent a menu of alternatives of some kind. The purpose of this paper is simply to evaluate menus as an appropriate model of competition among mechanism designers when there is only a single agent.

As will become apparent, menus are not universal in the sense of Epstein and Peters [4]. ${ }^{7}$ The universal set of mechanisms has the property that any arbitrary class of indirect mechanisms can be embedded into it. In this sense, the universal set extends any arbitrary set of indirect mechanisms. It creates new deviations that can destroy equilibrium relative to some arbitrarily re stricted set of indirect mechanisms. It also creates mechanisms that can be used to support equilibrium outcomes that cannot be supported relative to arbitrarily restricted sets of indirect mechanisms. The embeddings (in this universal class) associated with menus and alternative sets of indirect mech-

\footnotetext{
${ }^{5}$ For example, Bernheim and Whinston [1], Martimort and Stole [8]or Biias, Martimort and Rochet [2].

'It has been suggested to me that this is referred to as the 'taxation principle' although I am unsure of the source of this term.

${ }^{7}$ For the same reason, menus are not universal in the way that direct mechanisms are universal in the singlemechanism designer problem. Arbitrary indirect mechanisms cannot be uniquely associated with some speci- ${ }^{-}$menu.
} 
anisms will generally intersect, without any associated proper containment.

The di \pm culty is that the same menu o eered in di ßerent ways (that is to say, o eered as alternative indirect mechanisms) can induce di Berent continuation equilibria. To account for this, the universal set of mechanisms must be at least as large the set of all menus plus the set of all continuation equilibria associated with those menus. Menus al one cannot be used to describe more general indirect mechanisms. ${ }^{8}$

However, menus possess properties weaker than universality in common agency problems that are al most as good. The ${ }^{-}$rst theorem presented below shows that any equilibrium relative to some set of indirect medhanisms can betranslated into an equilibrium relative to some subset of the set of menus. ${ }^{9}$ This translation does not preserve payo ${ }^{\mathbb{S}}$ in the way that translations into the universal set of mechanisms does. However the translations of indirect mechanisms played along the equilibrium path do preserve payo \&s so that the equilibrium payo $\mathbb{B}$ relative to some set of feasible indirect mechanisms can be 'represented' as equilibrium payo®s relative to some restricted set of menus. This means that if there are models of indirect competition that have desirable properties, like robustness [4], then it must be possible to characterize the associated equilibrium payo®s as equilibrium relative to the set of menus.

This result does not imply that every equilibrium in menus is necessarily interesting. The set of menus is a strict subset of the universal set of mechanisms, so it is likely that some equilibrium relative to the set of menus will not be robust to the possibility that principals can deviate to more complicated mechanisms. This property is the key property of the universal set of mechanisms discussed in Epstein and Peters [4]. Equilibrium relative to the universal set of mechanisms will continue as equilibria no matter how the set of feasible mechanisms is extended. The second result in the paper is concerned with this issue.

\footnotetext{
${ }^{8}$ It might seem that all that this requires is that the seller o $\operatorname{Ber}$ a menu along with a suggestion about how the agent should choose from the menu (following Myerson). The di \pm culty with this approach is that the suggestion that the seller makes to theagent should depend on the agent's true type Since this type depends on the market information that the agent has, this immediately makes the problem as di \pm cult as trying to describe a universal set of mechanisms.

${ }^{9} \mathrm{As}$ will be seen, the menus that are needed are more complex than those that have traditionally been used in the literature
} 
Unfortunately menus do not have the strong robustness properties of equilibria relative to the universal set of medhanisms. However, they do have a weaker robustness property in the sense that for any extension of the set of mechanisms, it will always be possibleto construct a continuation equilibrium for the extension such that the payo®s from the original equilibrium are preserved.

\section{B asics}

There are $n$ sellers dealing with a single buyer. The terms buyer and seller need not be interpreted literally. This terminology is common in the literature on competing mechanisms ([9],[15], or [4]). The literature on common agency has been more creative in generating examples, for example multiple lenders dealing with a single borrower (Biias Martimort and Rochet [2] and Parlour and Rajan [13]), multiple regulators overseeing the operation of single ${ }^{-} \mathrm{rm}$ (Bernheim and Whinston [1]) or multiple lobbyists trying to in ${ }^{\circ}$ uence government policy (Dixit and Grossman [3]). The trading process begins when sellers simultaneously announce the mechanisms they plan to use An important assumption is that the buyer observes market information completely while no seller can directly observe the mechanism that has been o Bered by any other seller. After seeing the contracts o eered by each seller, the buyer communicates with sellers and take actions that a eect the payo ${ }^{R S}$ of all the sellers.

The primitives of the model are

A: space of 'simple' actions available to each seller

E : space of actions that the buyer carries out for the sellers

- : valuations space (including the 'usual type' of a buyer)

F : cdf according to which (all sellers believe) the buyer's valuation is drawn

To avoid measuretheoretic considerations, it will be assumed that the sets $A, E$ and - are all ' nite An element of the set $A$ is a complete description of the contractible part of the relationship between the seller and the buyer. This would include any transfer between buyer and seller and any action 
taken by the seller. Actions for the seller might indude contracts that specify contractible actions that the buyer has to take An important assumption is that sellers cannot write contracts contingent on the contract o Bers or actions of the other sellers. The set $\mathrm{E}$ describes the set of uncontractable actions available to the buyer. The set $\mathrm{E}$ might consist of a vector of e®orts that the buyer undertakes for each seller. Alternatively, in a competing mechanism problem, $E$ is just a list of all the sellers. The buyer's choice simply re ${ }^{\circ} e c t s$ the mechanism that the buyer chooses to participate in.

Buyers and sellers have expected utility preferences. Sellers' payo ${ }_{S}$ are represented by $v: A^{n} f E ; ![0 ; 1]$. For the buyer, payo $\mathbb{B}$ are represented by the function $u$ : $A^{n} £ E £-i$ ! [0;1]. To simplify some of the arguments below, it is assumed that there is an action a that each seller $\mathrm{j}$ can take such that for any $a_{j} 2 \mathrm{~A}$

$$
u\left(\underline{a} ; a_{i j} ; e_{i} !\right) \cdot u\left(a_{j} ; a_{i j} ; e_{i} !\right)
$$

for all $a_{i j} 2 A^{n_{i} 1}$; e $2 E$ and for any ! 2 - . This action is used at various points to prevent the buyer from sending messages the seller does not want to hear under any circumstances.

\subsection{Standard M odel of Competition}

For any measurable set $X$, Let $4(X)$ denote the set of probability measures on $X$. When a topology on $4(X)$ is needed, the topology of weak convergence is assumed. For any $x 24$ (X), let supp $x$ be the support of the distribution $\mathrm{X}$. Fix a measurable space of messages $C$ that the buyer can send to a seller. For simplicity it is assumed that this set of messages that the buyer can send to each seller is the same Let $R$ be the (measurable) set of responses that sellers can send back to the buyer. Both these message spaces are assumed compact metric, but beyond that are perfectly general in the degree and nature of the communication about the other sellers' mechanisms that they permit. For example, the messages could allow the buyer to communicate the mechanisms being used by other sellers. The messages could be contingent plans describing the way the buyer will communicate under di ßerent circumstances.

An indirect mechanism ${ }^{\circ}$ for seller $\mathrm{j}$ speci ${ }^{-}$es feasible messages, $\mathrm{C}$ and $\mathrm{R}$, as well as the probability distribution over actions and messages that the 
seller will use to respond to any message that the buyer sends him. So ${ }^{\circ}$ is a measurable map from $C$ into $4(A f R)$. With a slight abuse of notation, let ${ }^{\circ}=f^{\circ}{ }_{1}::{ }^{\circ} \mathrm{n}$ refer to the entire array of mechanisms o Bered by the sellers. For any particular element of $4(A f R)$, each report $r 2 R$ that is sent with positive probability induces a unique posterior belief about the probability with which the seller has chosen each action. This posterior probability lies in 4 (A). Since Bayes rule involves a measurable map from $R$ ! A, each mechanism can be associated with a measurable map from C into 4 ( $A$ f 4 (A)). In words, each message induces a joint distribution on actions and posterior beliefs. It will be convenient below to think of indirect mechanisms in this way. It will be assumed in the sequed that the image of each feasible ${ }^{\circ}$ is closed in 4 ( $f £ 4(A)$ ).

Denote by $i$ the set of feasible indirect mechanisms, endowed with some topology. The goal of this paper is to try to characterize 'good' models of indirect competition, i.e., to specify 'good' choices for i . Good models of indirect equilibrium should be such that equilibrium exists, so for the purposes of this paper, it is innocuous to assume that i has the 'usual' desirable properties, for example that it is compact metric.

The allocations are then determined by the following process. First, sellers simultaneously and publicly announce the mechanisms that they plan to use to determine their allocations. The buyer then communicates with each of the sellers by sending each of them one of the messages in the sets $C_{j}$ that they specify. The sellers respond and choose actions according to the ${ }^{\circ}$ that they have committed themselves to. Once the buyer sees the sellers' response to his message, he chooses his e®ort.

The simplest example might be price competition. Suppose that each seller has a single unit of output to o er and that the buyer wishes to purchase exactly one unit. Each seller announces a price. The buyer chooses one and only one seller as a potential trading partner after seeing the prices that the sellers have to o $\mathrm{Ber}$. In this case there is no communication, so that $\mathrm{C}$ and $\mathrm{R}$ are empty for every seller. The buyer's eßort is simply the seller that he selects, so that $\mathrm{E}$ is simply a list of all the sellers.

Buyer behavior in each medhanism depends on the buyer's valuation and on the mechanisms that he observes being o ered by the other sellers. A communications strategy is a measurable mapping $\in:-f^{n} ! 4\left(C^{n}\right)$ that describes the (probability distribution over) messages that the buyer will send to the sellers as a function of the buyer's type and the array of mechanisms 
that he is o eered by the sellers. A decision strategy 14a: - $f i^{n} f C^{n} f R^{n}$ ! $4(E)$ is a measurable mapping that describes the probability distribution the buyer will use to choose his action as a function of his type, the array of mechanisms that he has been o ßered, themessages he has sent, and the array of messages received from the sellers. The pair ( $; ; / A$ together constitute a continuation strategy for the buyer. Say that the continuation strategy (e; 7 Ais a continuation equilibrium if the buyer has no incentive to deviate from this strategy for any of his valuations; for any array of mechanisms opered by the sellers, or for any array of messages that he receives from the sellers.

Again, since $;$ is intended to represent a reasonable model of the compe tition among the sellers, it is natural to assume that continuation equilibria exist. It is not di \pm cult to construct models of indirect competition where continuation equilibrium do not exist (one mechanism might be - I will trade with the buyer who names the largest integer). ${ }^{10}$ It is also easy to think of models of indirect competition where sellers can oßer mechanisms that do not make sense (each seller o eers a price equal to the price oßered by the other seller). There is no need to worry about whether such models are good descriptions of competition between sellers - it is immediately apparent that they are not. Thus in the discussion that follows we restrict attention to indirect models that possess all the usual desirata of predictive models.

It should also be noted that when a particular continuation equilibrium is assigned, this does not involve or require that this continuation equilibrium be unique $A$ mechanism is of interest to a seller partly because of the continuation equilibrium that it delivers. Alternative equilibrium selections will beinterpreted as alternative models of indirect competition in this paper.

When we want to emphasize the underlying set of indirect mechanisms i werefer to $(\mathrm{e} ; \mathrm{A}$ as a continuation equilibrium relative to $\mathrm{i}$. When wewish to emphasize a particular array of mechanisms, we refer to $\left.\left(\in\left(\Phi^{\circ}\right) ; 1 / 44 \phi \zeta^{\circ}\right)\right)$ as a continuation equilibrium relative to ${ }^{\circ}$.

The key to the standard (one seller) revelation principle, is that composing a mechanism with buyers' strategies yields a mapping from valuations into actions, or in other words, a 'direct mechanism'. In the context of this

\footnotetext{
${ }^{10}$ In fact Myerson [12] gives an example in which the sellers equilibrium does not exist when sellers are constrained to use naive direct mechanisms in which the buyers report only their types.
} 
paper, there is a corresponding composition. Let

$$
m_{\varepsilon ; z_{4}}:-f i^{n} i ! 4\left(A^{n} f E\right) \text {, }
$$

be the joint distribution of actions and e®orts induced by a particular continuation equilibrium $(\because ;$; 4 .

These de nitions make it possible to describe the buyer's and seller's payo ${ }^{\text {s.s. Suppose that the competing }}{ }^{-} \mathrm{rms}$ choose the randomizations $\pm=$ $f_{1}::: \neq g$ with $\neq 24(i)$ for all $j=1::: n$, and that buyer behavior is described by the continuation equilibrium strategy $(e ; 7)$. The buyer's payo ${ }^{\circledR}$ depends on the mechanisms that sellers o eer. Let ${ }^{\circ}=f^{\circ}{ }_{1}:::^{\circ} \mathrm{ng}$ be any array of mechanisms. Then the payo ${ }^{\circledR}$ that the buyer gets is

$$
\begin{aligned}
& U_{i}\left({ }^{\circ} ; €, 14 !\right)= \\
& \text { z z } \\
& \text { фषৎ } u\left(a_{1}::: a_{n} ; e_{;} !\right) d m_{e_{i} ; z_{4}}\left(! ;^{\circ}\right)
\end{aligned}
$$

The seller who chooses the randomization $\neq$ expects the payo ${ }^{\circledR}$

$$
\begin{aligned}
& V_{i}\left(+; t_{j} ; e^{\prime} ; A^{\prime}\right. \\
& \text { Z Z }
\end{aligned}
$$

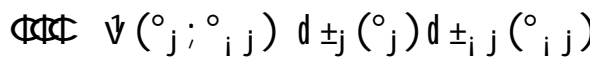

where

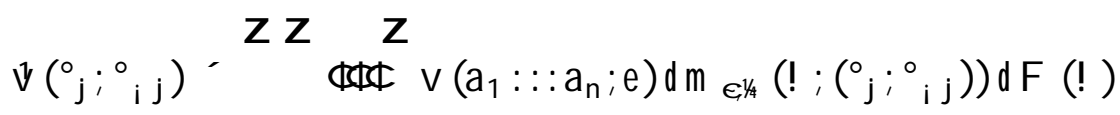

In an obvious notation, $d_{t_{j}}\left({ }^{\circ}{ }_{i j}\right)=f d_{z} ;::: d_{f_{i}} ; d_{f}::: d_{+} g$.

Say that $\left(e ; 1 / 4 ; ;:: t_{+}\right)$is an equilibrium relative to $(M ; i)$, or simply that $\left(i ; e^{\prime} ; 4+4:: t_{+}\right)$is an equilibrium for $M$, if: $(e ; 7 / 4$ is a continuation equilibrium and for each $\mathrm{j}=1 ;::: \mathrm{n}$ and

$$
\text { † } 2 \arg \max _{ \pm 2 \Phi(i)} V_{i}\left( \pm^{0} ; \Psi_{i j} ; e_{;} ; \not A\right. \text {. }
$$

It should be apparent from this that the actions that any particular seller takes, and the erort level that is induced depend not only on the buyer's valuation but also on the mechanisms that have been o Bered by the other - rms. It might seem reasonable to model common agency in the familiar way by having sellers choose allocations in response to the buyer's reports 
about preferences. As actions and e®orts will generally depend on mechanisms o eered by other ${ }^{-} \mathrm{rms}$, it should be clear that the allocations and eßort induced by somemodes of indirect competition will not be reproduciblewith this kind of direct mechanism. This was illustrated initially by the example in Peck [14]. A complete series of examples illustrating the pitfalls of this approach are given in Martimort and Stole [8] for the common agency case.

\section{M enus}

In the simplest single seller single buyer problem, any direct mechanism assigns an outcome to each report the agent makes about his type. No matter how complex the type space is for the agent, the range of this mapping obviously constitutes a menu of alternatives from which the agent can choose This works in reverse as well, since every menu of alternatives can be written as an incentive compatible direct mechanism that yields the same outcome It is natural to enquire whether this approach might work in the common agency problem when there is a single agent.

In it's simplest form, a menu is simply a mechanism with a message space equal to the set of actions $A$ along with a mapping ${ }^{\circ}:$ A ! A satisfying ${ }^{\circ x}$ (a) $2 \mathrm{fa}$; ag for all ! . The interpretation is that the mechanism ${ }^{\circ x}$ either gives the buyer the action that he requests, or gives the buyer $\underline{\text { a. }}$. The seller's message space $R$ is set equal to the empty set. This is the approach taken in [2] who allow the sellers to compete in non-linear pricing schemes. The action that the seller takes in that case consists of the monetary transfer and an asset trade.

In general, medhanisms formed by oßering menus of this kind will be restrictive since sellers might want to exploit buyer risk aversion by oæering random mechanisms in which the buyer is oßered a distribution over the set of outcomes for some of his types. If the seller does this, he might also want to send a random reply to the buyer that is correlated with the action that he chooses in order to maintain some connection between his action and the e®ort that the seller makes. Menus of the simple kind described above can not be used to reproduce this kind of behavior.

So to model menus in this more general setting suppose the seller uses the message space $R=4(A)$ and let $A^{x}=4(A \mp 4(A))$. The message that the seller sends in this case should be interpreted as a recommendation 
to the buyer about the posterior probability with which he should believe that the seller has taken the various actions. An action for the seller is then a joint distribution over simple actions and signals. In the formulation that follows the seller will o eer the buyer the choice from a menu of actions in $A^{x}$. For this reason, the set of messages $C$ available to the buyer will be equal to $A^{\star}$. Now de ne $i^{x}$ to be the set of all mappings ${ }^{\circ x}: A^{\star} ! A^{x}$ satisfying

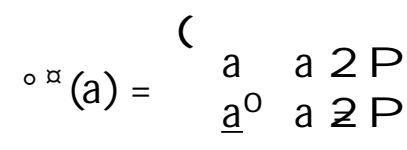

for some closed subset $P 1 / 2 A^{x}$, where $\underline{a}^{0}$ is a degenerate measure that assigns all probability weight to the outcome $\underline{a}$ and sends the degenerate message $\underline{a}$ with probability 1 .

The following theorem shows that arbitrary indirect medhanisms can be replaced with mechanisms in which sellers o eer the buyer a menu of alternatives (from $A^{x}$ ). This new menu is simply the set of alternatives that the buyer could have induced in the original direct mechanism by sending appropriate messages to the seller.

T heor em 1 Supposethat $;$ is some space of indirect mechanisms; let $(e ; 1 / 4+1 ;::$ : +4$)$ be an equilibrium relative to $i$. Then there is a map $\tilde{A}: ; \quad ; \quad i^{\text {" }}$, an array of randomizations $\left(t_{1}^{\alpha}::::_{n}^{\infty}\right)$ over $i^{\alpha}$ and a continuation equilibrium $\left(c^{\alpha ;} ;{ }^{1 / 4}\right)$ relative to $i{ }^{x}$ such that

1.

$$
U_{i^{\sharp}}\left(\tilde{A}\left({ }^{0}\right) ; C^{\alpha} ; 1 / \frac{\pi}{4} ; !\right)=U_{i}\left({ }^{0} ; \epsilon^{1 / 4} 4 !\right)
$$

for all ! and for all ${ }^{\circ} 2 i^{n}$; and

2. for each ${ }^{\circ} 2$ supp +

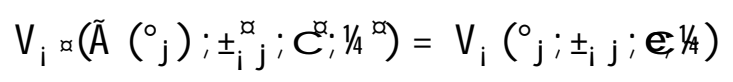

otherwise if ${ }^{\circ}{ }_{j} Z$ supp +

$$
V_{i}{ }^{a}\left(\tilde{A}\left({ }^{\circ}{ }_{j}\right) ; t_{i}^{\alpha} ; C^{\alpha} ; 1 / \alpha\right) \cdot V_{i}\left(+;+_{j} ; e ; 1^{1 / A}\right.
$$


Every equilibrium relative to i has a payo $₫$ equivalent representation in $i{ }^{\text {" }}$ where the representation is given by the mapping $\tilde{A}$. The representations are equivalent in the sense if all sellers mechanisms are translated into $i{ }^{\text {x }}$ the buyer gets the same payo ${ }^{\circledR}$ in the continuation equilibrium relative to these translated mechanisms as he does in the original continuation equilibrium. Furthermore sellers gets the same payo ${ }^{\circledR}$ from translated medhanisms as they receive in the initial equilibrium provided that they play one of their equilibrium strategies. Unpro table deviations for sellers in i correspond after translation into unpro table deviations relative to $\mathrm{i}^{\text {" }}$.

Thetranslation Ã is straightforward. It simply takes all of theactions that the buyer could have induced by sending some message to the seller, ${ }^{\circ} \mathrm{j}(\mathrm{C})$, and o eers this to the buyer as a menu of alternatives that he needs to choose from. The buyer's continuation strategy is then constructed to reproduce his behavior in the original continuation equilibrium. This preserves the payo ${ }^{\circledR S}$ of the various players.

The proof is mathematically trivial, but complex because of thefact (mentioned previously) that many di ßerent indirect mechanisms might translate into the same menu of alternatives. This would not be a problem, except for the fact that the buyer could conceivably choose from the menu and select an e An example is presented to illustrate this below.

This is the reason that o ${ }^{\circledR}$ equilibrium path mechanisms can not betranslated in a way that preserves theseller's payo ${ }^{\circledR S}{ }^{11}$ If the samemenu is o eered in two di ßerent ways in the original game, and the buyer chooses di ßerent e the original continuation equilibrium depending on which of the two (menu equivalent) indirect mechanisms that he o ers. . The translation into menus will require that the buyer respond with only one e®ort to both medhanisms. If the two indirect mechanisms both lie on the equilibrium path, this won't be a problem because they will generate the same payo®for the seller anyway. All that can be guaranteed $\mathrm{o} \circledast$ the equilibrium path is that the translated mechanism will yield a payo ${ }^{\circledR}$ that is no larger than the equilibrium path payo®in the original game.

The reader might wonder whether this problem could be resolved if the

\footnotetext{
${ }^{11}$ It bears mention here that the universal class of mechanisms described by Epstein and Peters [4] generates a transformation that preserves these payo $\mathbb{B}_{S} \mathrm{O} \otimes t$ the equilibrium path.
} 
seller simply extends the menu by o eering a message instructing the buyer how to select his erort (and how to choose from the menu). This is the approach that Myerson [11] used for the single principal problem. Indeed, this is why the revelation principle will normally require the principal to send a message to his agents recommending the actions they should take This approach is not pursued here, partly because it is unnecessary for the purposes of this paper, and partly because it raises much more di \pm cult problems in the multi-principal context. For example, the recommendations that the principal makes to the agent will generally have to depend on the agent's full type induding all his market information. To construct the appropriate messages mechanism needs to deal with the conceptually complex considerations associated with universal set of mechanisms as discussed in [4] - precisely the considerations that this paper is trying to avoid.

The second point to keep in mind is that equilibria for sellers relative to i will not generally translate into equilibria relative to $i{ }^{\text {x }}$. The reason is that i will typically incorporate various implicit restrictions on the mechanisms that sellers are allowed to use. This is expressed formally by observing that $\tilde{A}(i) \frac{1}{2} i^{x}$. This observation explains the various failures of the revelation principle that have been uncovered in the literature For example it is not hard to imaginethat if theimage of $i$ is 'small' enough in $i{ }^{\text {a }}$ then equilibrium outcomes relative to ${ }{ }^{\text {a }}$ will generate payo ${ }^{\circledR s}$ that cannot be attained by any combination of medhanisms in $i$. This is the basis of the example given in Peck [14]. Secondly, even if the payo ${ }_{\mathrm{BS}}$ in some equilibrium relative to $i^{\text {a }}$ are attainable by combinations of mechanisms in $i$, these payo $\&_{S}$ may not be supportable as equilibria relative to $i$. This is the basis of the example given in Martimort and Stole [8].

The third point to note is that the theorem given here is considerable weaker than the one in Epstein and Peters [4]. In that paper conditions are given under which every mechanism in $;$ (on or o®the equilibrium path) is translated into a payo ${ }^{\circledR}$ equivalent mechanism in $i^{x}$ (again the translation here is not payo $\circledast$ equivalent $0 \circledR$ the equilibrium path).

Despite the fact that menus in $A^{x}$ are relatively tractable, they are considerably more complicated than the menus that have been used in the literature Non-linear pricing schemes, for example, involve menus of simple actions, without any randomization of any messages from the sellers to attempt to in ${ }^{\circ}$ uence buyer behavior. The non-linear pricing problem with a single agent is simpler than the problem considered here if the buyer takes 
no uncontractable action on behalf of the seller. In this case, there is no need for the seller to send messages to the buyer to in ${ }^{\circ}$ uence his eßort, and the set of menus of elements from 4 (A) will be su \pm cient to prove a revelation principle similar to the one above

The required randomization means that this set of menus will still be more complicated than the simple non-linear pricing schemes that have been used in the literature. Optimal incentive schemes with risk aversion will often require the use of random outcomes. The typical approach is to assume that randomization is impossible. A previous version of this paper assumed that contractible randomization was di \pm cult enough to kep the set of feasible actions ( of either a random or non-random kind) ' nite. In this case, a version of the Theorem 1 can be proved in when sellers o eer only menus of simple actions.

\section{Example}

This section develops a simple example to illustrate the revelation principle proved above There are two sellers and a single buyer who can have one of two possible types $\mu_{1}$ or $\mu_{2}$ with equal probability. The sellers have three available actions called A, B, and C. The buyer does not make any e®ort and it is assumed impossible for the seller to write contracts that commit him to any speci ${ }^{-} \mathrm{c}$ randomization over these thre outcomes. ${ }^{12}$ The payoßs to seller 1 , seller 2 and the buyer respectively are listed in the following table.

\begin{tabular}{|c|c|c|c|c|c|c|}
\hline \multicolumn{3}{|c|}{$\mu_{\mathrm{l}}$} & \\
\hline A & B & C & & A & B & C \\
\hline $0 ; 0 ; 0$ & $2 ; 0 ; 2$ & i $1 ; 5 ; 3$ & A & 3; 3; 3 & $0 ; 0 ; 0$ & $0 ; 0 ; 0$ \\
\hline $0 ; 2 ; 2$ & $1 ; 1 ; 1$ & $0 ; 0 ; 0$ & B & $0 ; 0 ; 0$ & $1 ; 1 ; 1$ & $0 ; 0 ; 0$ \\
\hline $5 ;$ i $1 ; 3$ & $0 ; 0 ; 0$ & $0 ; 0 ; 0$ & C & $0 ; 0 ; 0$ & $0 ; 0 ; 0$ & $0 ; 0 ; 0$ \\
\hline
\end{tabular}

The ${ }^{-}$rst box is a variant of the example presented in Martimort and Stole [8]. ${ }^{13}$ There is an equilibrium for this problem in which both sellers o®er the

\footnotetext{
${ }^{12}$ This does not stop him, of course, from using a random strategy to choose among the three actions.

${ }^{13}$ They describe a menu equilibrium for this box in which each seller o $\mathbb{B}$ ers the menu $f B ; C g$ to the buyer who then chooses the option B from both. This is not an equilibrium if contractible randomizations are possible If they are either seller can pro tably deviate
} 
action $C$ to the buyer. This can be interpreted as an equilibrium in direct mechanisms, where each seller o eers a direct mechanism in which the action chosen is independent of the buyer's report. This is also an equilibrium in menus (in which each seller o Bers a degenerate menu).

If the competition is modelled by allowing sellers to o Ber menus of simple actions to the buyer, there is a relatively simple equilibrium in which each seller o eers the buyer the menu $\mathrm{fA} ; \mathrm{Cg}$. The buyer chooses $\mathrm{A}$ from both sellers when his type is $\mu_{2}$ and randomizes choosing $A$ from one seller and $C$ from the other with equal probability when his type is $\mu_{1}$. The buyer's payo ${ }^{\circledR}$ in this case is 3 whatever his type. Each seller gets 3 when the buyer is type $\mu_{2}$ and 2 (that is, $\frac{1}{2} 5 i \frac{1}{2}$ ) when the buyer is type $\mu_{1}$.

To show that this is an equilibrium in menus it is necessary to check that neither seller can pro'tably deviate by modifying the menu that he oßers. For example, consider the payo ${ }^{\circledR}$ that the seller can get by deleting theoption A from his menu. If the buyer has type $\mu_{1}$ he will choose $A$ from the other seller for sure and the deviating seller will get payo ${ }^{\circ} 5$. The downside is that the seller now receives payo ${ }^{\circledR} 0$ when the buyer is type $\mu_{2}$. This gives the seller's expected payo ${ }^{\circledR} a s 5=2$, exactly what it was in the initial equilibrium. It is straightforward to check that deviations to alternative menus are also unpro $^{-}$table ${ }^{14}$

Alternatively, considering modeling the competition by al lowing sellers to choose from a slightly larger set of contracts, consisting of all possible menus, and all di rect mechanisms (meaning that the buyer reports either $\mu_{1}$ or $\mu_{2}$ and the seller responds with one of the actions $A, B$, or $C$ ). Refer to the menu $\mathrm{fA} ; \mathrm{Cg}$ as the medhanism ${ }^{\circ \mathrm{m}}$ in this space. Then one deviation that the seller might consider is to o er a direct mechanism ${ }^{\circ d}$ which chooses the action $\mathrm{C}$ if the buyer reports $\mu_{1}$ and $A$ if the buyer reports $\mu_{2}$. Suppose that the buyer responds to this deviation by reporting $\mu_{2}$ to the deviator no matter what his

to a random mechanism (that is, a degenerate menu from $4(\mathrm{~A})$ ) that chooses action $A$ with small enough probability. I am indebted to Serge Severinov for pointing this out to me

${ }^{14}$ If contractible randomizations are possible, this menu will not be an equilibrium. A pro $^{-}$table deviation would involve o eering a menu consisting of the outcome $\mathrm{C}$ for sure, or a random outcome that provides $\mathrm{A}$ with high probability and $\mathrm{C}$ with complementary probability. If the buyer has type $\mu_{\mathrm{I}}$ he can no longer randomize, but is forced instead to choose the outcome $C$ for sure with the deviator. Since the option $A$ is still available, the buyer will choose it when he has type $\mu_{2}$. 
type is (so that the deviator al ways chooses A). Then, when the buyer's type is $\mu_{1}$ he chooses the action $C$ from the other seller's menu. This continuation equilibrium makes the deviation strictly unpro ${ }^{-}$table since the deviator loses any chance at the payo ${ }^{\circledR} 5$ that he gets with positive probability along the equilibrium path.

To translate ${ }^{\circ d}$ into the corresponding menu observe that if the other seller o ers the degenerate menu consisting only' of the action A than the buyer will report truthfully under ${ }^{\circ} \mathrm{d}$. Thus $\tilde{A}{ }^{\circ d}$ is just equal to the menu consisting of the options $f A ; C g$, obviously the same as $\tilde{A}\left({ }^{\circ} \mathrm{m}\right)$. The same menu of options can be o Bered in two distinct ways, and the buyer responds to these o eers di Berently. This di ßerence makes the sellers pro't strictly lower when he o Bers ${ }^{\circ d}$ than when he o eers ${ }^{\circ} \mathrm{m}$ in the original game. Then when the mechanism ${ }^{\circ d}$ gets translated into $i^{\text {a }}$ the payo ${ }^{\circledR}$ that the seller gets from the translation is strictly higher than the payo ${ }^{\circledR}$ he got from the deviation in the original equilibrium. Nonetheless the payo ®is no larger than his equilibrium payo ${ }^{\circledR}$ as speci ${ }^{-}$ed by Theorem 1.

This example has another property. Turn the argument around and imagine starting with the set of menus and extending the set of medhanisms available to the seller to include direct mechanisms. Whether the sellers will continue to o $\mathrm{Ber}$ the menu $\mathrm{fA} ; \mathrm{Cg}$ depends on how they expect buyers to respond.. If the buyer is expected to report truthfully when the seller de viates to the direct mechanism ${ }^{\circ d}$, then the equilibrium in menus will fall apart since the deviating seller will then ensure the payo ${ }^{2} 5$ for himself when the buyer has type $\mu_{1}$. This illustrates that the equilibrium in menus is not robust in the sense of Epstein and Peters [4].

This leads nicely into the second theorem in the paper. There is a continuation equilibrium that could be assigned to the deviation that will break up the equilibrium, but there is also one that will preserve it. The theorem in the next section shows that this is generally true with common agency.

\section{W eak R obustness}

The wide use made of direct mechanisms (or menus) in single mechanism designer problems stems from two facts - " $r s t$, direct mechanisms are simple. Perhaps more important, once sellers ${ }^{-}$nd the best direct mechanism they can be sure that they cannot improve their pro ts any more by experimenting 
with other morecomplex mechanisms. Neither menus, nor direct mechanisms have this property in competing mechanism problems. This is illustrated in the example given above. These same examples suggest an alternative, since the ability of the seller to increase his pro ${ }^{-}$ts by o ering more complicated mechanisms seems to depend on the way that the continuation equilibrium is assigned. Some assignments increase pro ${ }^{-} \mathrm{ts}$, but there al ways seems to be an alternative assignment that does not increase the seller's pro ${ }^{-} \mathrm{ts}$. It turns out that when there is a single agent, the property is true more generally. The point of this section is to illustrate this idea - if sellers are constrained to use menus of the kind described above, then there will al ways be a way to assign the continuation equilibrium so that this equilibrium persists when the set of mechanisms is enlarged.

To illustrate this idea consider two feasible sets of indirect mechanisms i and $i^{1}$. Say that $i^{1}<i \quad\left(i^{1}\right.$ is bigger than $\left.i\right)$ if there exists an embedding $\mathbb{R}^{Q}: \mathrm{i}$ ! $\mathrm{i}^{1}$. This idea is natural, there are more mechanisms in $\mathrm{i}^{1}$ than in $i$. Now take two modes of competition with feasible sets of mechanisms $i$, and $i^{1}$ and such that $i^{1}<i$. Let the associated continuation equilibria be $\left(\epsilon ; 1 / 4\right.$ and $(\epsilon ; 1 / 4)$ respectively. As before, let $m_{e ; z_{4}}\left(\Phi^{\circ}\right)$ and $m_{e^{1} ; Z_{4}}\left(\zeta^{\circ 1}\right)$ be the joint distributions on actions and eßort induced by these continuation equilibria when the array of mechanisms on o $B e r$ are ${ }^{\circ}$ and ${ }^{\circ 1}$ respectively. The continuation equilibrium $\left(i^{1} ; \epsilon ; ; / 4\right)$ is said to extend $(i ; \epsilon ; A)$ if there is an embedding ${ }^{\circledR}: \mathrm{i}$ ! $\mathrm{i}^{1}$ such that for all ${ }^{\circ}$ in $\mathrm{i}^{\mathrm{n}}$

$$
m_{E_{i} ; z_{4}}\left(\Phi^{\circ}\right)=m_{\epsilon^{1} ; Z_{4}}\left(\Phi \circledast\left(^{\circ}\right)\right)
$$

The generalization of the idea behind direct mechanisms in the single mechanism designer problem is to imagine sellers exploring more complex mechanisms (in $\mathrm{i}^{1}$ ) that are not envisioned by the 'ad hoc' model of competition speci ed in the economic model of competition (i.e, i ). A sensible model of competition is one that has the property that the equilibrium allocations that it describes do not disappear when new, more complicated mechanisms are added to the sellers' feasible sets.

D e $e^{-}$nition 2 (Strong R obustness) An equilibrium ( $i ; \epsilon_{i} 1 / 4 \pm$ is said to be strongly robust if for any extension $\left(i^{1} ; \epsilon ; 1 / 4\right)$ for which $i^{1}$ is compact

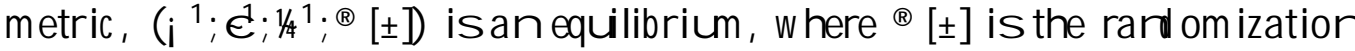
on $\mathrm{i}^{1}$ induced by \pm and $\AA$. 
Strongly robust equilibria are analyzed in Epstein and Peters [4]. As shown in the example above, equilibria in menus are not strongly robust. If menus have desirable properties at all they must be weaker than full robustness.

D e- nition 3 (W eak R obustness) An equilibrium $(i ; \in ; 4 \pm$ is said to be weakly robust if for every compact metric $\mathrm{i}^{1}<\mathrm{i}$ there exists an extension $\left(i{ }^{1} ; \epsilon^{1} ; 74\right)$ such that $\left(i^{1} ; \epsilon^{1} ; 1+4 ; \circledR[\exists)\right.$ is an equilibrium, where $\mathbb{B}[\exists$ is the randomization on $\mathrm{i}^{1}$ induced by \pm and $\mathbb{R}$.

This lead to our second result

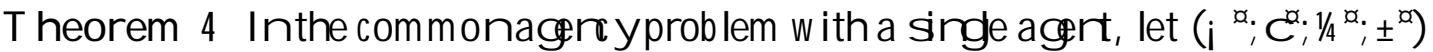
be an equilibrium. Then $\left(i^{x ;} ; c^{\alpha ;} ; 1 / 4 ; \pm^{x}\right)$ is weakly robust.

To see the idea behind the proof, it might help to consider the case where there is only a single mechanism designer. For any arbitrary mechanism - that this seller o eers from $\mathrm{i}$, the image ${ }^{\circ}(\mathrm{C})$ is the 'menu' of alternative actions that the buyer can choose from. Typically therewill be one (or more) mechanisms ${ }^{\circ}$ in $\mathrm{i}$ that o $\mathrm{Ber}$ the buyer the same menu of alternatives as $\circ 2{ }^{\circledR}$. Typically these will yield theseller a di ßerent payo®than the payo ${ }^{\circledR}$ he gets from ${ }^{\circ}$. The reason for this is that the continuation equilibrium will induce the buyer to send di Berent messages and take di eerent actions than he does when he sees ${ }^{\circ x}$. This is precisely why the seller might like thelarger set of mechanisms. Yet since the menu of actions and eßorts available to the buyer is the same when he is o Bered ${ }^{\circ}$ as it is when he is o eered ${ }^{\circ x}$, the maximum payo $\circledast$ that he can attain from both mechanisms must be the same. This means that a new continuation equilibrium can be constructed by having the buyer choose from the 'menu' associated with ${ }^{\circ}$ in exactly the same way as he does when he chooses from the menu ${ }^{\circ x}$. Then, in this new continuation equilibrium, the seller must get the same payo $₫$ when he o eers ${ }^{\circ}$ as he does when he o eers ${ }^{\circ x}$. With this construction, every payo ${ }^{\circledR}$ that the seller can attain in i coincides with a payo ${ }^{\circledR}$ that he could have attained in $i^{\text {x. }}$. It follows immediately that whatever was optimal for the

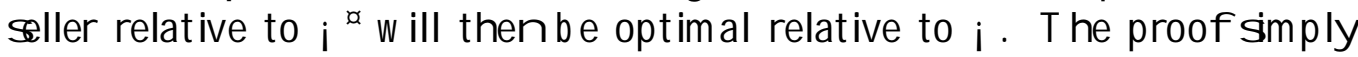
extends this reasoning to the case where there are many sellers. 


\section{Conclusion}

In the case where many principals try to control the incentives of a single agent, the competition among them can be erectively modelled by having them o eer alternative menus to agents. This model will be 'eßective' in two ways. First, equilibria from arbitrary compeitive models can be represented as equilibria relative to (a subset of) the set of menus. Secondly, equilibria relative to the set of all menus are weakly robust. So, equilibria in menus need not fall apart when the set of medhanisms is extended.

Despite the fact that common agency is now a common way of modelling incentive problems, an obvious shortcoming of the analysis in this paper stems from the fact that there is only a single agent. Unfortunately, the methods presented here do not suggest any extension to the multiple agent case. Even if there is only a single principal, menus oßer little advantage with multiple agents, because the choice from the menu is a 'joint' choice (of all the agents) not an individual choice A key part of the argument in Theorem 1 relies on the fact that when a single agent faces the same choice set o eered in two di ßerent ways, his optimal choice from it will always give him the same payo ${ }^{\circledR}$ - even when this choice varies with the way the choice set if o eered. When multiple agents are o eered the same normal form game in di Berent ways, it is quite possible that the equilibrium that they choose (and consequently every agents payo $\otimes_{\text {) }}$ will vary. Oncethe principal is required to send instructions to the agents about how to play a game, an in ${ }^{-}$nite regress emerges - the principal's recommendation about how to play should depend on whether the other principals' recommendations depend on whether the principal's recommendation depends: : : and so on. It is possible to deal with this (see [4]) but the appropriate set of medhanisms is complex.

\section{A ppendix - Proofs}

We begin with some basic de nitions and relationships. For any subset $P \quad 1 / 2$ $A^{x}$ de $^{-}$ne- $(P): A^{x}$ ! $A^{x}$ such that

$$
-(P)(a)=\begin{array}{ll}
a & \text { if a } 2 \text { P } \\
\underline{a}^{0} & \text { otherwise }
\end{array}
$$


In words, ${ }^{-}(P)$ is a function that responds to any joint distribution (action) in $\mathrm{P}$ with the same joint distribution (over actions and messages), otherwise it responds with $\underline{a}^{0}$ (recall this is a degenerate distribution that assigns all probability weight to the pair ( $\underline{a} ; \underline{a})$ ). With this understanding, we will refer to ${ }^{-}(P)$ as a menu of alternatives from $A^{x}$.

Each mechanism ${ }^{\circ}$ and report c produces a joint distribution over simple actions and messages for the seller. Each message that the buyer receives induces some posterior belief in 4 (A) about the simple action that the seller has undertaken. Thus each report that the buyer makes to the seller induces a pure action in $A^{x}$, $4(A \mp 4(A))$. With a slight abuse of notation, de ne the set of actions (in $A^{x}$ ) that the buyer can induce by sending some message to the seller as ${ }^{\circ}$ (C) $1 / 2 A^{x}$. Recall that this set is closed in $A^{x}$ by assumption.

The buyer chooses his eßort after observing the message associated with the action $a^{x} 2 A^{x}$. Together, the sellers' strategies \pm the continuation strategies ( $\epsilon^{1}$ A $A$ and the buyer's type! induce a joint distribution over $\left(A^{x}\right)^{n} f$ E.

$D e^{-}$nition 5 For any $D 1 / 2 i^{n}$ such that the $\pm(D)>0$ de ne $b(! ; D)$ to be the joint distribution on $\left(A^{x}\right)^{n} f E$ induced by the equilibrium $(i ; i ; 1 / 4 \pm$ conditional on the sel lers' mechanisms being drawn from the subset $D \frac{1}{2} i^{n} \cdot{ }^{15}$ Write $b^{A x}(! ; D)$ as the marginal distribution of $b$ on the space of actions, $\left(A^{x}\right)^{n}$.

Write (a; q) as a typical element of $A \_4$ (A). Sincethebuyer's posterior beliefs are de ned beforeheis forced to choosean eßort, the utility associated with any particular array of actions $a^{a} 2\left(A^{x}\right)^{n}$ is given by

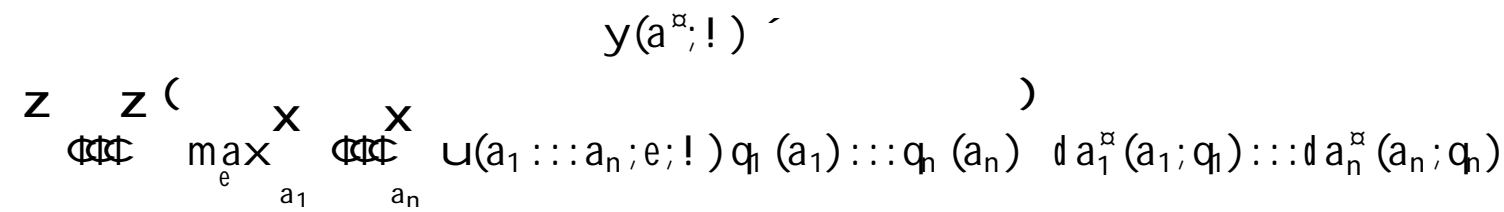

With this notation, the buyer's payo ${ }^{\circledR}$ in any continuation equilibrium can be written as

$$
\mathrm{U}_{\mathrm{i}}\left({ }^{\circ} ; \mathrm{E} ; 14 !\right)=
$$

\footnotetext{
${ }^{15}$ The fact that this distribution depends on $\epsilon_{1} 1 / 4$ and \pm is suppressed to ease notation.
} 


$$
\begin{aligned}
& { }^{Z} \stackrel{Z}{Z} u\left(a_{1}::: a_{n} ; e ; !\right) d m_{\epsilon_{i} ; z_{4}}\left(! ;{ }^{\circ}\right)= \\
& \text { z } \mathrm{z} \\
& \text { ФФ屯 fy }\left(a_{1}^{a \alpha} ;::: a_{n}^{a} ; !\right) g d b^{A x}\left(! ;{ }^{\circ}\right)= \\
& \max ^{n} y\left(a_{1}^{a} ;::: a_{n}^{x} ; !\right): a_{j}^{a} 2^{\circ}{ }_{j}(C) 8 j=1::: n^{\circ}
\end{aligned}
$$

The ${ }^{-}$rst equality follows from the fact that every e®ort level that the buyer takes with positive probability must maximize his interim payo ${ }^{\circledR}$ The ${ }^{-}$nal equality follows from the fact that $b$ is the distribution associated with a continuation equilibrium following ${ }^{\circ}$. The use of max instead of sup in the - nal operation results from the assumption that a continuation equilibrium al ways exists.

\subsection{Proof of Theorem 1}

P r o of. To begin, the map Ã is described, and the strategies that sellers and the buyer use in the continuation equilibrium relative to the new space of mechanisms are described. De ${ }^{-}$ne the transformation $\tilde{A}_{j}$ from $i$ into $i{ }^{\alpha}$ as

$$
\tilde{A}_{j}:{ }_{j}^{\circ} \nabla !{ }_{j}^{o p}\left(\Phi={ }^{-}\left({ }^{\circ}(C)\right)(\emptyset\right.
$$

This transformation simply converts a medhanism ${ }^{\circ}$ into the menu of alternatives in $A^{\alpha}$ that it provides. Le $t^{\alpha}$ be the measure induced by + on $i^{\alpha}$ by this map.

$\tilde{A}_{j}$ will generally be a many to one mapping with inverse correspondence $\tilde{A}_{j}^{i}\left(\phi\right.$. Choose an arbitrary selection $\hat{A}_{j}^{i}{ }^{1}(\Phi$ from this correspondence. For any collection $f_{1}^{\circ x}:::_{n}^{\circ x} g^{1 / 2} i^{n}$ de ne the sets

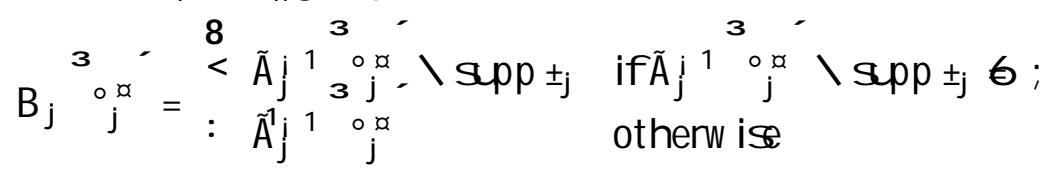

We can now specify the continuation equilibrium relative to $i{ }^{x}$. Begin with the case where ${ }^{\circ x} z \tilde{A}(i)^{n}$. By the de nition of $i^{x}$, The buyer must choose a distribution over actions from each seller that lies in a closed subset of $A^{x}$, as well as an eßort from a ' nite set. Since the buyer's payo®s are linear (by expected utility) with respect to the distribution of actions that sellers choose, they are continuous. It follows that a best choiceaction pair from each menu exists for the buyer. So it will always be possible to assign 
some continuation equilibrium to the array of mechanisms. Since arrays like this will play no role in what follows, the continuation equilibrium can be assigned in any convenient fashion.

On the other hand if $\left.{ }^{\circ x}=f_{1}^{\circ x} ;:::_{n}^{\circ x} 2 \tilde{A}(i)\right)^{n} d e^{-} n e$

$$
C^{a}(! ; \circ x)=b^{A^{x}}\left(! ; f B_{1}\left(\begin{array}{c}
\circ x \\
1
\end{array}\right) ;::: B_{n}\left(\begin{array}{c}
\circ x \\
n
\end{array}\right) g\right)
$$

where $b^{A x}\left(! ; f B_{1}\left(\begin{array}{c}o x \\ 1\end{array}\right) ;::: B_{n}\left(\begin{array}{c}o x \\ n\end{array}\right) g\right)$ denotes the marginal distribution on $\left(A^{x}\right)^{n}$ induced by $b\left(! ; f B_{1}\left(\begin{array}{c}o x \\ 1\end{array}\right) ;::: B_{n}\left(\begin{array}{c}o x \\ n\end{array}\right) g\right)$.

To de ne the buyer's e®ort choice, let $a^{\alpha}=f a_{1}^{\alpha} ;::: a_{n}^{\alpha} g$ denotethe buyer's actual choice from the menu o Bered by each seller, and let q 24 (A $)^{n}$ bethe array of messages that the buyer receives. Note that the buyer's posterior beliefs (after seeing the sellers' messages) also lie in $4(A)^{n}$. The buyer chooses his e®ort level according to

$$
1 / \frac{a}{4}\left(! ;{ }^{\circ a} ; q\right)=b^{E}\left(! ; f B_{1}\left(\begin{array}{c}
o d \\
1
\end{array}\right) ;::: B_{n}\left(\begin{array}{c}
o x \\
n
\end{array}\right) g j a^{a} ; q\right)
$$

where $b^{E}\left(! ; f B_{1}\left(\begin{array}{c}o x \\ 1\end{array}\right) ;::: B_{n}\left(\begin{array}{c}o x \\ n\end{array}\right) g j a^{\alpha} ; q\right)$ is the distribution of eßort induced by $b\left(! ; f B_{1}\left(\begin{array}{c}o x \\ 1\end{array}\right) ;::: B_{n}\left(\begin{array}{c}\circ 口 \\ n\end{array}\right) g\right)$ conditional on the sellers' actions being given by $a^{a}$ and the buyer's posterior beliefs being given by $q$.

The next step is to show that this strategy constitutes a continuation equilibrium, and that the buyer's payo ${ }^{\circledR}$ when he follows this continuation strategy in the transformed problem is the same as his payo®in the original problem. To begin, consider the payo ${ }^{\circledR}$ that the buyer receives in the continuation equilibrium relative to $\left({ }^{\circ} ; c^{\alpha} ; 1 / \frac{1 / 4}{4}\right)$ after choosing the array $a^{a}$ from the various sellers menus, and receiving the array of messages $q$ from the sellers. From (8), this is given by

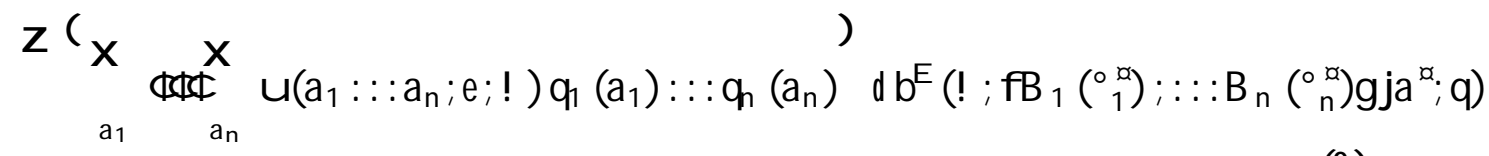

Consider any action $a_{j}^{a}$ in the menu o eered by seller $j$. By construction, each such action must coincide with a joint distribution of simple actions and posterior beliefs that the buyer can induce (by sending appropriate messages) to the sel ber in the continuation equilibrium relative to $\left({ }^{\circ}{ }_{j}{ }^{\circ}{ }_{i} j ; i_{i} ; A\right.$ for any ${ }^{\circ}{ }_{j} 2 B_{j}{ }_{j}^{\circ o}$. It follows that any message $q$ that the buyer could receive when he selects $a_{j}^{a}$ from seller $j^{9} s$ menu must be equal to the correct posterior for a conditional on that message. Then from the properties 
of the continuation equilibrium ( $;, \mathrm{A} A$ every eBort leve in the support of $\mathrm{b}^{\mathrm{E}}\left(\right.$ ! ; $\left.f \mathrm{~B}_{1}\left(\begin{array}{c}\mathrm{ox} \\ 1\end{array}\right) ;::: \mathrm{B}_{\mathrm{n}}\left(\begin{array}{c}\mathrm{ox} \\ \mathrm{n}\end{array}\right) \mathrm{gja} \mathrm{a}^{\mathrm{a}} ; q\right)$ must maximize the buyer's expected payo ${ }^{\circledR}$ conditional on the posterior belief q. Thus (9) can be simpli ${ }^{-}$ed to

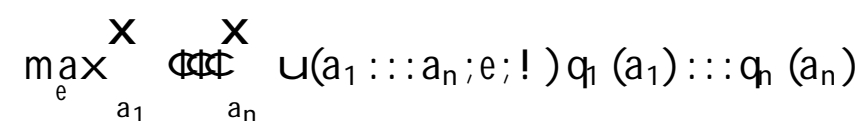

Then the ex ante payo ${ }^{\circledR}$ associated with the choice $a^{\alpha}$ is just

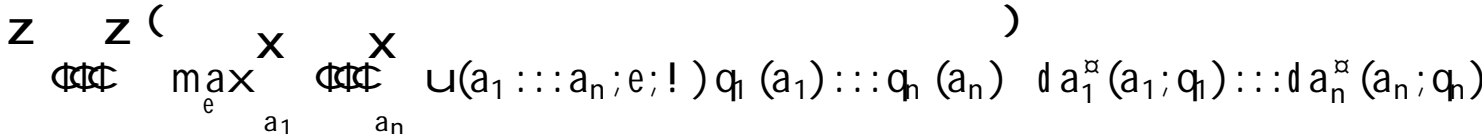

$$
\begin{aligned}
& =y\left(a^{\alpha} ; !\right)
\end{aligned}
$$

The buyer selects according to the distribution $b^{A^{x}}\left(! ; f B_{1}\left(\begin{array}{c}o x \\ 1\end{array}\right) ;::: B_{n}\left(\begin{array}{c}o x \\ n\end{array}\right) g\right)$. So this strategy yields the payo ${ }^{\circledR}$

$$
\begin{aligned}
& Z \\
& y\left(a^{a} ; !\right) d b^{x}\left(! ; f B_{1}\left(\begin{array}{c}
o x \\
1
\end{array}\right) ;::: B_{n}\left(\begin{array}{c}
o x \\
n
\end{array}\right) g\right)
\end{aligned}
$$

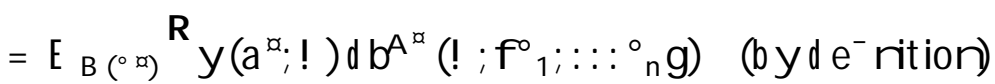

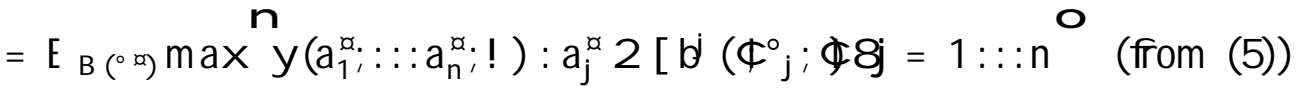

$$
\begin{aligned}
& =\max { }^{n} y\left(a_{1}^{a} ;::: a_{n}^{a} ; !\right): a_{j}^{a} 2\left[b j \left(\varphi^{\circ} ; \phi \varnothing j=1::: n^{\circ}\right.\right.
\end{aligned}
$$

(because of the fact that $\left[b\left(\phi_{j}^{\circ} ; \Phi\right.\right.$ is the same for all $\left.{ }_{j}^{\circ} 2 B_{j}{ }_{j}^{o d}\right)$. Since the choice sets in this last expression are the ones o eered in the menus associated with the mechanisms in ${ }^{\circ x}$, this line veri ${ }^{-}$es that the continuation strategy speci ${ }^{-}$ed for the buyers is a continuation equilibrium. Finally applying (5) once again gives this payo ${ }^{\circledR}$ equal to

$$
=U_{i}\left({ }^{0} ; \epsilon_{i}, 44\right)
$$

which establishes the ${ }^{-}$rst property in the theorem.

Finally, it remains to show that

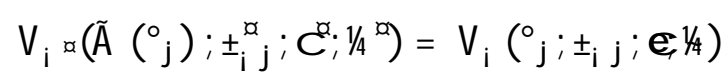

for all ${ }^{\circ} 2$ supp + . 


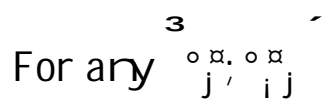

Z Z

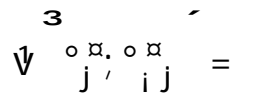

$v\left(a_{1}::: a_{n} ; e\right) d b\left(! ; f B_{1}\left(\begin{array}{c}o d \\ 1\end{array}\right) ;::: B_{n}\left(\begin{array}{c}o p \\ n\end{array}\right) g\right) d F(!)$

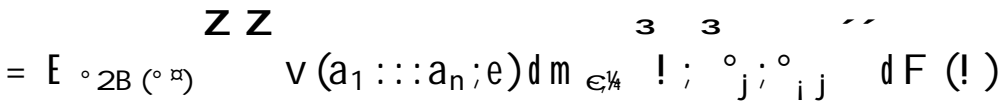

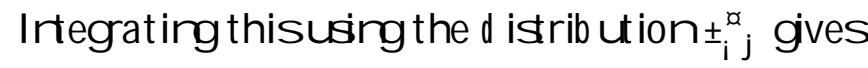

$$
\begin{aligned}
& V_{i}{ }^{\infty}\left({ }_{j}^{\alpha} ; \pm_{j}^{\alpha} ; C^{\alpha} ; 1 / \alpha\right)
\end{aligned}
$$

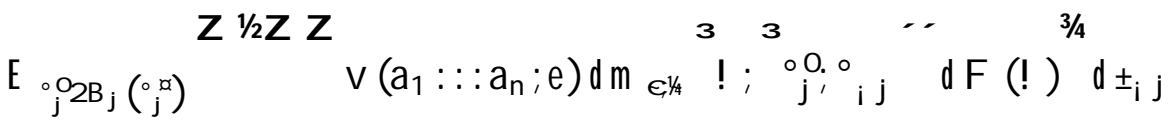

$$
\begin{aligned}
& =E_{j}^{\circ 02 B_{j}\left({ }_{j}^{\circ a}\right)} V_{i}\left({ }_{j}^{\circ 0} ; 土_{j} ; e ; 1 / A\right.
\end{aligned}
$$

If ${ }_{j}^{\circ x}=\tilde{A}\left({ }^{\circ}\right)$ for some ${ }_{j}^{\circ} 2$ supp + , then by the de $e^{-}$ition, each ${ }^{\circ}{ }_{j} 2 B_{j}{ }^{\circ x}$ is an element of the support of + . Since + is an equilibrium distribution for

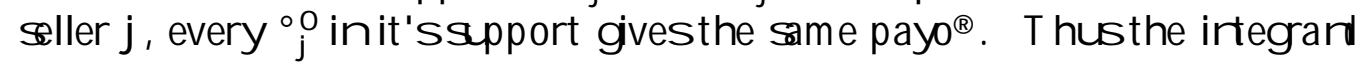
in this last expression is constant and equal to

$$
V_{i}\left({ }^{\circ} ; t_{i j} ; e^{\prime} \not A 4\right.
$$

as required.

Alternatively if $\tilde{A}^{i}{ }^{1} \underset{j}{o x}={ }^{\Phi} Z$ supp + then

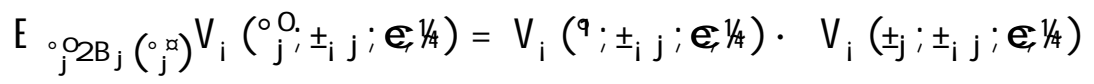

which proves the theorem.

\subsection{Proof of theorem 4}

P roof. Let $\left(i ; \epsilon_{1 / 4}\right.$ be an extension of $\left(i^{\alpha} ; c^{\alpha ; 1 / \frac{\alpha}{4}}\right)$ with embedding given by $\AA$ The method of the proof is to convert deviations that lie outside of the range of $₫$ into the menus that they do provide, and then change the continuation equilibrium associated with those menus to coincide with the original equilibrium.

Use the transformation Ã from Theorem 1 to associate with each mechanism $^{\circ}{ }_{j} 2$ i the corresponding menu ${ }_{j}^{o x}(\phi)={ }^{-}\left({ }^{\circ}(C)\right)(\phi$. Now let 
$D\left({ }^{\circ} ;:::_{n}^{\circ}\right) 1 / 24\left(A^{n} f E\right)$ be the set of joint distributions over simple actions and eßorts that can be induced with some distribution of messages and some erort choice strategy, given the mechanisms $f^{\circ}{ }_{1} ;:::_{n}^{\circ} \mathrm{g}$. If the buyer if o ered an array of mechanisms $f^{\circ}{ }_{1} ;:::^{\circ} \mathrm{n}$ in the continuation equilibrium relative to $i$, he can induce any array of actions $f a_{1} ;::: a_{n} g$ in the set ${ }_{1}^{\circ}(C) f::: f_{n}^{\circ}(C)^{\prime}{ }_{1}^{o x}\left(\Phi f:::_{n}^{\circ x}\left(\Phi 1 / 2\left(A^{x}\right)^{n}\right.\right.$ by sending appropriate messages to the sellers. It follows that $D\left({ }^{\circ} ;:::_{n}^{\circ}\right)=D\left({ }_{1}^{\circ} ;:::_{n}^{\circ}\right)$. Since

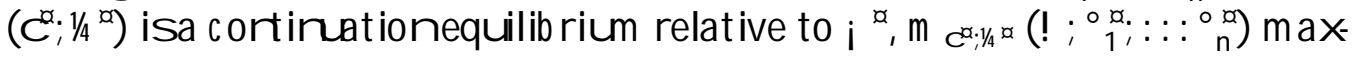
imizes the expected utility over $D\left(\begin{array}{c}o x, \\ 1\end{array} ;:: \begin{array}{c}\circ x \\ n\end{array}\right)$ of a buyer of type ! . It follows immediately that this distribution maximizes the buyer ! 's expected utility over the set $D\left({ }^{\circ}{ }_{1} ;:::_{n}^{\circ}\right)$. Choose any continuation strategy $e^{\circ}$ and $18{ }_{4}^{\circ}$ relative to i that satis ${ }^{-} e s$

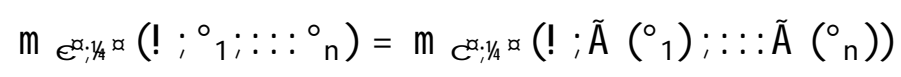

(this strategy exists by the de nition of $D)$. SinceD $\left({ }^{\circ}{ }_{1} ;:::{ }^{\circ}{ }_{n}\right)=D\left(\tilde{A}\left({ }^{\circ} 1\right) ;::: \tilde{A}\left({ }^{\circ} n\right)\right)$ this continuation strategy must be a continuation equilibrium.

Now we begin with an equilibrium $\left(t_{7}^{*} ;::: t_{n}^{*}\right)$ relative to $i^{\text {a }}$ with $\mathbb{R}\left[ \pm^{\infty}\right]$ as the induced distribution relative to $i$. The payo ${ }^{\circledR}$ to some seller who

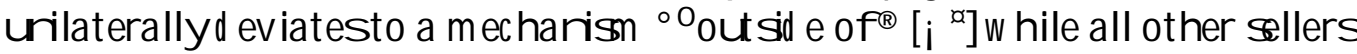
o eer medhanisms ${ }^{\circ} \mathrm{j}$ in the support of $\pm^{*}$ is given by

$$
\begin{aligned}
& \text { Z Z Z }
\end{aligned}
$$

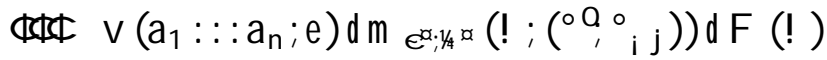

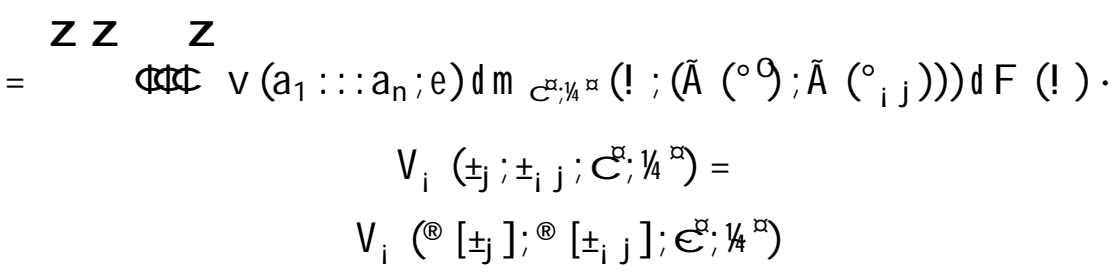

which implies that the equilibrium $\left(i^{x} ; \pm c^{\alpha} ; 1 / \frac{\pi}{4}\right)$ is weakly robust.

\section{R eferences}

[1] D. Bernheim and M. Whinston. Menu auctions, resourceallocations and economics in ${ }^{\circ}$ uence. Quarterly J ournal of Economics, 101:1\{31, 1987. 
[2] Bruno Biais, David Martimort, and J ean-Charles Rochet. Competing mechanisms in a common value environment. Technical report, Universite de Toulouse, 1997.

[3] Avinash Dixit, Gene Grossman, and Elhanan Helpman. Common agency and coordination: General theory and application to government policymaking. jpe, pages 752\{769, 1997.

[4] L. Epstein and M. Peters. A revelation principle for competing mechanisms. University of Toronto, 1997.

[5] A. Gibbard. Manipulation of voting schemes. Econometrica, 41:587\{601, 1973.

[6] J . Green and J.-J . Laßont. Characterization of satisfactory mechanisms for the revelation of preferences for public goods. Econometrica, 45:427\{ 438, 1977.

[7] Michael Katz. Game playing agents: Unobservable contracts as precommitments. mimeo, University of California at Berkeley, 1987.

[8] D. Martimort and L. Stole Communication spaces, equilibria sets and the revelation principle under common agency. mimeo University of Chicago, 1997.

[9] Preston McAfee Mechanism design by competing sellers. Econometrica, 61(6):1281\{1312, November 1993.

[10] Roger Myerson. Incentive compatibility and the bargaining problem. Econometrica, 47:61\{73, 1979.

[11] Roger Myerson. Optimal auction design. Mathematics of Operations Research, 6:55\{73, 1981.

[12] Roger Myerson. Optimal coordination mechanisms in generalized principal agent problems. J ournal of Mathematical Economics, 10:67\{81, 1982.

[13] Christine A. Parlour and Uday Rajan. Compeition in loan contracts. Technical report, Carnegie Mellon University, 1997. 
[14] J ames Peck. Competing mechanisms and the revelation principle Ohio State University Manuscript, page 12, 1995.

[15] Michael Peters. On the equivalence of wal rasian and non-walrasian equilibria in contract markets. Review of Economic Studies, pages 241 \{265, 1997. 